\section{Impact of mechanical and microstructural properties of potato puree-food additive complexes on extrusion-} based 3D printing

\author{
Iman Dankar ${ }^{\# \&}$, Montserrat Pujolà ${ }^{\&}$, Fawaz EL Omar ${ }^{\#}$, Francesc Sepulcre ${ }^{\&}$, Amira Haddarah ${ }^{\#}$ \\ "Lebanese University, Doctoral School of Science and Technology, EDST, Hadath, Lebanon \\ ${ }^{\&}$ Departament d'Enginyeria Agroalimentària i Biotecnologia, Universitat Politècnica de Catalunya. \\ BarcelonaTECH
}

\title{
Abstract
}

This paper studies the applicability of extrusion-based 3D printing for constructing novel shapes from potato puree and the effects of four additives (agar, alginate, lecithin and glycerol) added separately at three concentrations $(0.5$, $1,1.5 \%)$ on the internal strength, mechanical properties, microstructure and color of potato puree. The printability of the potato puree and the mixtures was assayed by examining the consistency of the extrusions and the stability and accuracy of the printed patterns. The results indicate that better printing was achieved at a nozzle height of $0.5 \mathrm{~cm}$ and a nozzle diameter of $4 \mathrm{~mm}$, with concentrations of alginate and agar between $0.5-1.5 \%$ and $0.5-1 \%$, respectively, providing the best printability and end-product stability, which was attributed to their respective high mechanical characteristics and specific mechanical energy (SME) values. Scanning electron microscopy (SEM) revealed that more convolutions were induced in the potato puree upon the addition of agar or alginate, which increased the puree stability. Three-dimensional printing did not significantly affect the surface color parameters of the final product. This study showed that the 3D printing process is a critical factor for initializing the production of customized healthy products.

1 Keywords: Texture, Scanning Electron Microscopy (SEM), Color, Specific Mechanical Energy (SME), 3D printing

2 Corresponding author: Dr. Montserrat Pujolà

E-mail: $\underline{\text { montserrat.pujola@upc.edu }}$

Postal address: 
Campus del Baix Llobregat UPC

C. Esteve Terradas, 8 EdificI D4

08860 Castelldefels, SPAIN

\section{Introduction}

There is a growing demand for the development of customized food for specialized dietary needs, such as products for athletes for recovery after training or products for expectant mothers that vary nutrient component levels by reducing amounts of undesirable ingredients and enhancing the presence of healthy ones (e.g., protein, vitamins, fiber). Moreover, elderly people who are facing physiological changes that occur with aging such as dysphagia and decreased sensory perception require special nutritive meals. Nevertheless, pureed food is delivered to them in an unappealing and unappetizing way. Children are another group of people who require special dietary intake. Children are more willing to consume healthy and nutritious snacks if they are presented in an innovative and fun way (Dankar, Haddarah, Omar, Sepulcre, \& Pujolà, 2018). However, the development of such customized foods must be conducted in a very precise and inventive way, which is where the role of 3D printing appears.

Three-dimensional food printing is an innovative technique that is of great potential interest and is continuously under debate for both consumers and food scientists due to its broad array of uses (Severini, Derossi, Ricci, Caporizzi, \& Fiore, 2018). The application of 3D food printing could be summarized as the ability to provide customized food to certain groups of people (de Roos, 2013) and to automatically generate a specific code to adjust composition, density or structure to the preferences and needs of the user. Moreover, 3D printing has demonstrated some interesting applications for industry by enhancing efficiency through the consolidation of multiple steps or even entire food production processes (Bak, 2003; Sun et al., 2015). For instance, the PepsiCo company decided to incorporate 3D printing in the manufacturing of its potato chips to save money and create healthier food after suffering serious problems in the sales of sugary drinks and fatty snacks (Simon, 2015). Extrusion printing through a syringe nozzle is the most popular technique employed because of its ability to process the widest array of foods, such as printing with mashed potatoes (Southerland, Walters, \& Huson, 2011), chocolates (Hao et al., 2010), cookie dough (Lipton et al., 2010), soft cheeses (Le Tohic et al., 2018), hydrogels and fibers (Lille, Nurmela, Nordlund, Metsä-Kortelainen, \& Sozer, 2017; Wang, Zhang, Bhandari, \& 
Yang, 2018) and blends of fruits and vegetables (Severini et al., 2017), and if coupled with more than one syringe, this technique can provide an infinite number of combinations of and a high degree of freedom for

52 foods.

53 On the other hand, important factors should be taken into consideration when extrusion printing. Maintaining compatibility between specific printing parameters and the corresponding printed substance is crucial to ensure high feasibility for 3D printing. The essential process parameters that can be modulated are the printing speed, the distance between the nozzle and the printing bed and the nozzle size; these are critical criteria that influence the final resolution of the constructed shape (Hao et al., 2010; Zhuo, 2015;Derossi, Caporizzi, Azzollini, \& Severini, 2018). Additionally, monitoring the properties and composition of the food material itself (ingredient rheology, electrical conductivity, density, textural quality, and physiochemical and microstructural properties) is imperative and aids in predicting the behavior of a particular food during 3D printing and in assembling a complex shape with many layers that is stable enough to maintain its profile for a long time post-deposition ( Dankar et al., 2018; Godoi, Prakash, \& Bhandari, 2016; Periard, Schaal, Schaal, Malone, \& Lipson, 2007; Yang, Zhang, Bhandari, \& Liu, 2018). Potato purees, now considered part of the nutritious ready-to-eat food market, could be combined with hydrocolloids that interact with potato starches in an attempt to improve the overall product quality and facilitate processing (Shi \& BeMiller, 2002). Therefore, scrutinizing the effects that certain food additives have on the starch structure and textural characteristics is important, because these effects affect the functionality of the whole food product. and their concentrations on the mechanical and microstructural properties of potato puree, to evaluate the feasibility of the substances for 3D printing, to characterize the printing process parameters, such as the distance on the superficial color of the final products. 
Commercial potato powder and whole milk were purchased from the local supermarket. Agar-agar, soy bean lecithin, sodium alginate and glycerol (food-grade) were procured from Sigma-Aldrich Co. The potato puree samples were prepared according to the following procedure: $450 \mathrm{~mL}$ of milk and $50 \mathrm{~mL}$ of water were first heated to $40^{\circ} \mathrm{C}$, and then, $115 \mathrm{~g}$ of commercial potato powder was added. The mixture was then homogenized using an electrical hand blender (Braun, Germany). The same procedure was followed for preparing the puree samples with the different additives at concentrations of $0.5,1.0$ and 1.5\% (Shi \& BeMiller, 2002). Additives were added at quantities corresponding to the desired concentrations to the warmed solution (milk and water) prior to the incorporation of the potato powder. However, for the agar samples, the solutions were boiled to $100^{\circ} \mathrm{C}$, and the dehydrated potato was then added. All prepared puree samples were placed in an incubator and held at a temperature of $20^{\circ} \mathrm{C}$ preceding any measurements.

87

\section{Extrusion Parameters and Determination of Specific Mechanical Energy}

To optimize the 3D printing process, the effects of additives (agar, alginate, glycerol and lecithin), applied speed $\left(1,2\right.$ and $\left.4 \mathrm{~mm} \cdot \mathrm{s}^{-1}\right)$ and extruder hole diameter $(3$ and $5 \mathrm{~mm})$ on the extrusion process were studied using a TA.XT Plus Texture Analyzer (Stable MicroSystems, Godalwig. UK) device with a 50-kg cell load. of materials in the extrusion process (Guerrero, Beatty, Kerry, \& De La Caba, 2012). Potato puree samples with and without additives were carefully scooped into acrylic cylinders to a height of $35 \mathrm{~mm}$. The extrusion process was carried out by locking the distance traveled by the compression disc along the cylinder to $20 \mathrm{~mm}$. For each extruder hole diameter ( 3 and $5 \mathrm{~mm}$ ), speeds of 1,2 , and $4 \mathrm{~mm} \cdot \mathrm{s}^{1}$ were applied. The weight collected in $\mathrm{kg}$ and

97 the force $\left(\mathrm{kg} \cdot \mathrm{ms}^{-2}\right)$ applied during extrusion was measured. The SME was then calculated using the following formula:

\section{Mechanical characteristics}


The mechanical characteristics of the additives alone at different concentrations $(0.5,1$ and $1.5 \mathrm{~g}$ of additive in $100 \mathrm{ml}$ of distilled water) and after being added to the potato puree were tested, including the firmness, consistency and cohesiveness, using the aforementioned TA.XT Plus Textural Analyzer coupled with a back extrusion cell and a $35 \mathrm{~mm}$ disc. Samples of potato puree up to $40 \mathrm{~mm}$ high were placed in a standard-size cylinder. During the test, the disc penetrated a distance of $30 \mathrm{~mm}$ at a speed of $2 \mathrm{~mm} \cdot \mathrm{s}^{-1}$, after which the probe returned to the original position. The peak in the positive area is taken as the measurement of firmness $(\mathrm{kg})$. The area under the curve up to this point is defined as the consistency (kg.s). The maximum negative force is taken as an indication of the cohesiveness (kg) (Angioloni \& Collar, 2009). Each sample was tested at least 5 times.

\section{Scanning Electron Microscopy (SEM)}

Scanning electron microscopy (SERON SCI2100) was used to determine the surface structures of all the puree samples, which were first subjected to vacuum in a vacuum chamber to be dehydrated to avoid swelling in the microscope. Samples were then mounted on circular aluminum stubs with double-sided adhesive tape and coated with $20 \mathrm{~nm}$ of gold prior to observation. The SEM experiments were carried out at $15 \mathrm{kV}$ and $4.0 \mathrm{~K}$.

\section{Color Measurements of Potato Puree Samples}

To evaluate the color properties of the puree samples, a MINOLTA tristimulus colorimeter CR-400 $a^{*}, b^{*}$ of the samples were measured, and the chroma $\left(\mathrm{C}=\left(\mathrm{a}^{2}+\mathrm{b}^{*}\right)^{1 / 2}\right.$ (saturation) and hue angle $(\mathrm{H}=\arctan$ $\left(b^{*} / a^{*}\right)($ matrix color)) were calculated. The color measurement values presented are the means of 6 tests performed before and after 3D printing.

\section{D Food Printing conditions}

A RepRap BCN3D+ printer (designed by CIM Foundation) coupled with a syringe tool (100 mL volume and 4 

object is transferred through an SD card from a CAD program (CURA 15.02.01). Speeds set in the CURA program were as follows: travel speed $=100 \mathrm{~mm} \cdot \mathrm{s}^{-1}$, infill speed $=40 \mathrm{~mm} \cdot \mathrm{s}^{-1}$, printing speed $=40 \mathrm{~mm} \cdot \mathrm{s}^{-1}$, flow $\%=100$ and retraction speed $=40 \mathrm{~mm} \cdot \mathrm{s}^{-1}$.

\section{Statistical Analysis}

Statistical analyses of the data were conducted on Minitab 18 (Minitab lnk. Conventry, UK). Data concerning SME, textural characteristics and color assessment were tested for significant differences $(p<0.05)$ using analysis of variance, one-way ANOVA and Tukey's HSD comparison test.

\section{Results and Discussion}

\section{Effects of the Extrusion Parameters on Specific Mechanical Energy values of potato puree} extruder hole caused an increase in the SME of the samples due to higher acquired friction during extrusion, which necessitates an increase in the applied force (Table 1). Thus, a larger hole diameter $(5 \mathrm{~mm})$ facilitated extrusion with proper ordering of the layers. Moreover, significant differences were seen in the SME exerted at various extrusion speeds at a fixed extruder hole diameter; the SME and the extrusion speed were found to be inversely proportional, where the highest value for the SME was recorded at the lowest speed and gradually

\section{Effects of additives on specific mechanical energy value of potato mixtures}

Table 2 shows the comparison between the potato puree and the potato purees with concentrations of $1 \%$ of 
speed of $2 \mathrm{~mm} \cdot \mathrm{s}^{-1}$. The SME value for the potato puree decreased significantly when $1 \%$ glycerol or lecithin was added (Table 2). This decrease could be attributed to the ability of glycerol and lecithin to retain moisture via the destabilization of the internal microstructure of the starch granules, therefore softening the material in accordance with Dankar et al., (2018) and Guerrero et al., (2012). Conversely, the addition of $1 \%$ alginate or agar in potato puree significantly increased the SME compared with the potato puree alone (Table 2). This result could be due to the tendency of hydrocolloids (agar or alginate) to form a continuous network of entanglements with starch molecules upon their addition to potatoes, leading to higher tensile strength and hardness and requiring a greater force to push the material out of an extruder (Fang, Zhang, \& Wei, 2015).

The characterization of the mechanical properties of food is important and aids in assessing the behavior of the food during processing and consumption. The mechanical characteristics of the food additives alone and at the three 175 different concentrations were first measured to understand the effects of the additives on the potato puree. The 176 results showed that the mechanical strength of the agar additive was significantly different $(\mathrm{p}<0.05)$ from the other 177 additives used in this work. On the other hand, the mechanical characteristics of the glycerol, lecithin and alginate 178 additives showed no significant differences when the concentrations were changed from 0,5 , to 1 and to $1,5 \%$, 179 whereas significant differences were detected in the mechanical properties of the agar measured at the different concentrations (Table 3). 
181 The firmness, consistency and cohesiveness of the potato puree alone and the potato purees with the additives are summarized in Figure 1. The results of statistical analyses showed no significant differences $(p<0,05)$ between the

183 firmness, cohesiveness and consistency of the potato puree and the purees with lecithin or glycerol at concentrations of $0.5,1.0$ and $1.5 \%$. The addition of glycerol or lecithin to potato puree promotes more swollen starch granules (Dankar et al., 2018) with a wider spread in the particle size distribution, giving rise to low values for firmness, cohesiveness and consistency (Afoakwa, Paterson, Fowler, \& Vieira, 2008) since these additives have emulsifying effects and the ability to lessen the structural integrities of foods such as waxy maize starch, cocoa spread cream, cassava starch and dark chocolate (Afoakwa, Paterson, Fowler, \& Vieira, 2009; Souza et al., 2012 Koushki \& Azizi, 2015; Yang et al., 2016). On the other hand, the addition of alginate or agar significantly increased the mechanical values of the potato puree, with this elevation being enhanced when the concentrations of the additives were higher. However, the only significant difference in the consistency and cohesiveness between the agar and alginate samples was obtained at the concentration of $1 \%$, which was marked by a higher consistency (Fig. 1). This behavior is attributable to the conveyed network structure that occurs between polysaccharide chains and the large-sized long additive molecules (agar or alginate) within the matrix and to the enhancement of the particle-particle surface contact (Huang, Kennedy, Li, Xu, \& Xie, 2007; Dankar, et al. 2018). Similar mechanical strength results are obtained when carboxy-methyl cellulose, xanthan or carrageenan are added to sweet potato puree, whipped cream and carrots, respectively (Truong \& Walter, 1994; Zhao, Zhao, Yang, \& Cui, 2009;Sharma et al., 2017). The alginate alone showed mechanical property values similar to that of the glycerol and lecithin, but when the alginate was incorporated in the potato puree, the resulting mixture had high mechanical property values comparable with that of the agar.

This difference could be related to the interaction of the alginate with the calcium ions abundantly present in the

202 milk and the potatoes used in the preparation of the samples, which consequently enhanced the textural strength and 203 viscoelastic properties of the puree as also reported by Truong et al. (1995) and Fasina et al. (2003). On the other hand, the agar solely formed a gel that, upon interaction with other molecules, formed a more complex entangled network, which enhances its thickening ability (BeMiller, 2011; Milani \& Maleki, 2012). 
Thus in terms of mechanical strength, the greatest strengthening effect exerted by the agar and the alginate on the potato puree allows for products with the sufficient mechanical integrity to support a built-up layered geometry without deformation, in contrast to those with glycerol and lecithin.

\section{Scanning Electron Microscopy (SEM)}

The SEM micrographs highlighted clear microstructural differences between the different puree samples. At $0.5 \%$, the puree sample with lecithin was comparable to the potato puree alone but had a more cotton-like texture, whereas more noticeable changes in the potato puree were detected with the additions of the glycerol, structures (Fig. 2b1, d1). However, this network-like structure was more compact in the sample with glycerol, which could be due to the ability of glycerol to enter the interior of polysaccharide chains and disrupt inter- and intra-molecular hydrogen bonds, making the polymer more elastic (Mali, Sakanaka, Yamashita, \& Grossmann, 2005). An expanded network with tiny wrinkles on the surface was produced by the agar. As the concentration of the agar increased, these tiny wrinkles evolved into a continuous phase with more folding and convolutions (Fig. 2d1, d3), which was the result of intense interactions between the starch and the agar (Phan, Debeaufort, Luu, \& Voilley, 2005), agar gel formation and agar-agar interactions at higher concentrations (Dankar et al., 2018). Therefore, a firmer and more complex network of interactions was seen in the structure of the puree with $1.5 \%$ agar, as revealed in the figures.

Similarly, the folding formed upon the addition of $0.5 \%$ alginate could be attributed to the formation of alginate-cation-polysaccharide complexes (Truong et al., 1995). When the concentration of alginate was increased to $1 \%$, a more consistent and firm structure was formed. This result reflects the characteristic mechanical behavior of alginate; at concentrations of $1 \%$, significant differences were detected between the agar and alginate samples, with a higher consistency value for the alginate, compared with a more cohesive structure for the agar that was expressed through higher folding formation. At $1.5 \%$ alginate, internal folding and convolutions were observed within the structure. The addition of additives at higher concentrations results in a greater availability of reactive sites and hence, increases their mode of functionality (Chen, Dickinson, Langton, \& Hermansson, 2000). In fact, these convolutions largely explain the increase in the internal strength and mechanical characteristics of potato puree upon the addition of agar or alginate. In contrast, when the 
concentration of lecithin in the potato puree was increased, a smooth surface with tiny pores was produced (Fig. $2 \mathrm{c} 1, \mathrm{c} 2)$. This behavior was ascribed to the two internal modes of action of lecithin. First, as regards the starch structure, lecithin can penetrate the starch molecules and induce modifications within the internal amyloseamylopectin and amylopectin-amylopectin bindings (Dankar et al., 2018). Consequently, more water molecules are able to penetrate the starch granules, leading to a more swelled starch structure that promotes the smoothness observed in the SEM figures (Fig. 2c1). Second, the emulsification properties of lecithin promoted the assembly of fine droplets that are an indication of a uniformly dispersed structure inside the food matrix (Afoakwa et al., 2009; Koushki \& Azizi, 2015). Likewise, increasing the concentration of the glycerol induced a similarly smooth surface comparable to that of the potato puree. These factors explain the absence of significant differences between the mechanical characteristics of the potato puree alone and those for potato puree with glycerol or lecithin added. The microstructures of the potato puree samples combined with the textural data provide vital input for the 3D printing process, since formation of strong networks like those displayed in the samples with agar or alginate additions could be used to yield integrated shape-retention properties with stabilizing effects.

\section{D printing Conditions for potato puree and potato puree with additives} distance from the nozzle to the printed bed was $\geq 1 \mathrm{~cm}$, the flow of material was irregular due to delayed deposition, and the layers extruded were breakable and incompatibly attached to the previous layers for all the puree samples. After many trials, the critical nozzle height for high-quality printed potato purees was determined to be $0.5 \mathrm{~cm}$. Similar results were obtained by Wang et al. (2018) and Hao et al. (2010) when printing surimi and chocolate gels, respectively; they found that the nozzle height critically affects the final geometry of the product.

The second optimization was the nozzle diameter, which directly affects the surface roughness and precision of printed objects (Yang et al., 2018). Because the 3D printer and the textural analyzer have different nozzle diameters, using the same diameter for both tests was impossible. This difference was minimized by using similar sized diameters, in both cases in the same range: 3 and $5 \mathrm{~mm}$ in the case of textural analyzer and 2 and $4 \mathrm{~mm}$ for the 3D printer. 
Using a $2 \mathrm{~mm}$ nozzle, printing with the potato puree and the potato purees with additives produced poorquality products in which the layers did not overlay with one another properly, and the shape was not wellmaintained, leading to a poor product mainly because the thin filament size that was extruded was not large enough to support the desired final structure for the potato puree. Whereas when a $4 \mathrm{~mm}$ nozzle was used, all the puree samples showed better printing quality. This result validates what was hypothesized while determining the extrusion parameters and SME values, where extrusion with the larger diameter size of $5 \mathrm{~mm}$ provided better layer organization than extrusion with a $3 \mathrm{~mm}$ diameter nozzle (refer to the SME results). A 4 $\mathrm{mm}$ nozzle is sized within the range of these two values and hence, the $2 \mathrm{~mm}$ nozzle was excluded. The critical nozzle diameter is specific to the particular type of food extruded, as has been stated by several authors (Hao et al., 2010; Yang, Zhang, Bhandari, \& Liu, 2018). prepared, the potato purees with the agar or alginate at the different concentrations tested were able to be printed in stable structures with many built-up layers that held their shape for a long time without collapsing (Fig. $3 \mathrm{a}$ and $3 \mathrm{~b}$, puree with $0,5 \%$ alginate and potato puree alone, respectively). This result could be directly attributed to the high internal strengths, demonstrated by the highest values measured for the textural properties (firmness, consistency, cohesiveness) and the high SME values exhibited by the purees with agar or alginate; the incorporation of gums into mashed potatoes has reportedly generally increased their resistance to deformation (Liu, Zhang, \& Bhandari, 2018). Furthermore, the stabilization of the final shapes printed with the purees with the alginate or agar increased with increasing additive concentration in the potato puree, except for the $1.5 \%$ agar, which displayed high SME and mechanical values compared with the other additives and in which the sample was more solid-like, retarding the process of printing.

The potato puree and the purees with glycerol or lecithin showed different behavior, in which printing a multiple-layered 3D structure started well with a smooth flow of potato paste (Fig. 3d). Nevertheless, when the structure reached its final stage, the many layers that were printed collapsed into each other (Fig. 3e), resulting in a poorly defined and deformed product, due to the low firmness, consistency and internal stability possessed by these samples, which confirms the previous results concerning the shape stability of the extruded layers 
from the texturometer. Conversely, these materials behaved well during the printing of flat structures with few layers. Thus, the stability of the final product depends not only on the substrate properties but also on the targeted geometry shape to be printed. The effect of the printed substrate on the quality of the final product has been reported by several authors. Yang et al. (2018) and Liu et al. (2018) observed that the addition of potato starch in certain concentration ranges in lemon juice and mashed potatoes, respectively, increased the viscosity of the printed substrate and therefore, ensured the delivery of more stable end-products. These results confirm that alginate and agar serve as better additives in food technological applications like 3D printing.

\section{Characteristics of the Final 3D Printed Products}

The color surface parameters for the puree samples, including the Luminosity, Chroma and hue angle, are dependent on the particulate distribution, absorptivity and scattering coefficients (Hutchings, 2011). Each food additive used had a different effect on the surface color of the potato puree due to their distinct effects on the starch structure and the distribution of the particles and their respective arrangements. Only the alginate and agar produced significant differences $(\mathrm{p}<0.05)$ in the luminosity parameters of the potato purees, with decreases in their values (Fig. 4), which could be attributed to alterations of the starch globule sizes and morphologies. Additionally, solely the agar exhibited an effect on the hue angle of the puree by elevating the level. The glycerol and lecithin produced significant differences in the Chroma of the potato purees by decreasing the saturation property, which could be ascribed to changes in the starch granule morphologies and sizes and the starch internal networks (Dankar et al., 2018). However, Afoakwa et al., (2008) reported that the addition of lecithin did not affect the luminosity, Chroma or hue angle of dark chocolates.

Generally, increasing the concentration of the additives in the potato puree did not cause any significant differences in the luminosity or hue angle. However, increasing the concentration of lecithin to $1.5 \%$ produced a significant difference in the Chroma of the potato puree by further decreasing the degree of saturation (Fig. 4). This result could be attributed to the lecithin (at $1.5 \%$ ) producing increased modifications of the internal starch granule interactions, yielding a dull surface appearance (less saturated).

On the other hand, Le Tohic et al. (2018) found that the printing process affected the surface color of printed cheeses, inducing a small decrease in the luminosity in contrast to our work, where the 3D printing process had no significant effect on any of the color parameters studied for all the puree samples. Thus, the 3D printing 

and companies. elderly and those facing swallowing and mastication problems, enhancing their appetites with safe, novel and nutritious foods (Aguilera \& Park, 2016). Table 4 presents the firmness values for all the puree samples in $\mathrm{kPa}$, which were converted according to the following formula:

The accessible range of consumption for elderly or people facing mastication problems is within the firmness value range of 20 to $40 \mathrm{kPa}$ (Serizawa et al., 2014), and all the tested puree samples fit well within this

\section{Conclusion}

Alginate (from $0.5 \%$ to $1.5 \%)$ and agar $(0.5$ and $1 \%)$ were the additives that provided more stability for printed products with corresponding increases in specific mechanical energy (SME).

The mechanical characteristics of firmness, consistency and cohesiveness showed significant differences $(\mathrm{p}<0.05)$ after the addition of agar or alginate to potato purees, and the effect was greater at higher 
concentrations. Nevertheless, when not mixed with potato puree, only agar had a significant difference in

346 mechanical characteristics among the additives.

The SEM figures demonstrate the different microstructural characteristics within the potato puree samples, wherein lecithin produced a cotton-like structure, alginate produced more folding, glycerol induced a more continuous network-like structure due to its ability to disrupt the inter- and intra-network interactions between the polysaccharide chains, and agar induced more folding and convolutions, which complements the textural value results.

The best extrusion conditions for the 3D-printed potato purees were achieved with a nozzle size of $4 \mathrm{~mm}$ and a critical nozzle height of $0.5 \mathrm{~cm}$ using a printing substrate of potato puree mixed with alginate $(0.5$ to $1.5 \%)$ or agar $(0.5$ and $1 \%)$ to provide the finest resolution of stable end-products with many built-up layers.

The optimal mechanical characteristic values for obtaining good quality $3 \mathrm{D}$ printed potato purees with additives fall within the following ranges: a firmness between 0.94 and $2.10 \mathrm{~kg}$, a consistency between 11.6 and $26.5 \mathrm{~kg} \cdot \mathrm{s}$ and a cohesiveness between 0.9 and $2.1 \mathrm{~kg}$. The color of the final product is not affected by the 3D printing process and all the printed samples showed good firmness values that fit well within the range of the maximum lingual pressure $(20-40 \mathrm{kPa})$, thus enabling potato puree or other foods to be used in innovative designs to produce a good substitute for the unappealing meals available for people facing mastication problems.

\section{Acknowledgements}

Authors wish to thanks the CIM foundation for providing the BCN 3D+ printer and Dr. Roland Habchech for the use of the SEM.

\section{References}

Afoakwa, E. O., Paterson, A., Fowler, M., \& Vieira, J. (2008). Particle size distribution and compositional effects on textural properties and appearance of dark chocolates. Journal of Food Engineering, 87(2), 181-190. https://doi.org/10.1016/j.jfoodeng.2007.11.025

Afoakwa, E. O., Paterson, A., Fowler, M., \& Vieira, J. (2009). Microstructure and mechanical properties related to particle size distribution and composition in dark chocolate. International Journal of Food Science \& Technology, 44(1), 111-119. https://doi.org/10.1111/j.1365-2621.2007.01677.x 
372

373

374

375

376

377

378

379

380

381

382

383

384

385

386

387

388

389

390

391

392

393

394

395

396

397

Aguilera, J. M., \& Park, D. J. (2016). Texture-modified foods for the elderly: Status, technology and opportunities. Trends in Food Science and Technology, 57, 156-164. https://doi.org/10.1016/j.tifs.2016.10.001

Angioloni, A., \& Collar, C. (2009). Small and large deformation viscoelastic behaviour of selected fibre blends with gelling properties. Food Hydrocolloids, 23(3), 742-748. https://doi.org/10.1016/j.foodhyd.2008.04.005

Bak, D. (2003). Rapid prototyping or rapid production? 3D printing processes move industry towards the latter. Assembly Automation, 23(4), 340-345. https://doi.org/10.1108/01445150310501190

BeMiller, J. N. (2011). Pasting, paste, and gel properties of starch-hydrocolloid combinations. Carbohydrate Polymers, 86(2), 386-423. https://doi.org/10.1016/J.CARBPOL.2011.05.064

Chen, J., Dickinson, E., Langton, M., \& Hermansson, A. (2000). Mechanical Properties and Microstructure of Heatset Whey Protein Emulsion Gels : Effect of Emulsifiers. Lebensm.-Wiss. u.-Technol. Academic Press, 33(4), 299-307. https://doi.org/10.1006/fstl.2000.0656

Dankar, I., Haddarah, A., El Omar, F., Sepulcre, F., \& Pujolà, M. (2018). Assessing the microstructural and rheological changes induced by food additives on potato puree. Food Chemistry, 240. https://doi.org/10.1016/j.foodchem.2017.07.121

Dankar, I., Haddarah, A., Omar, F. E. L., Sepulcre, F., \& Pujolà, M. (2018). 3D printing technology: The new era for food customization and elaboration. Trends in Food Science \& Technology, 75, 231-242. https://doi.org/10.1016/J.TIFS.2018.03.018

de Roos, B. (2013). Personalised nutrition: ready for practice? Proceedings of the Nutrition Society, 72(01), 48-52. https://doi.org/10.1017/S0029665112002844

Derossi, A., Caporizzi, R., Azzollini, D., \& Severini, C. (2017). Application of 3D printing for customized food. A case on the development of a fruit-based snack for children. Journal of Food Engineering, 220, 65-75 https://doi.org/10.1016/j.jfoodeng.2017.05.015

Fang, Y., Zhang, B., \& Wei, Y. (2015). Effects of the specific mechanical energy on the physicochemical properties of texturized soy protein during high-moisture extrusion cooking. Journal of Food Engineering, 121, 32-38. https://doi.org/10.1016/j.jfoodeng.2013.08.002

Fasina, O. O., Walter, W. M., Fleming, H. P., \& Simunovic, N. (2003). Viscoelastic properties of restructured 
sweetpotato puree. International Journal of Food Science and Technology, 38(4), 421-425. https://doi.org/10.1046/j.1365-2621.2003.00711.x

Godoi, F. C., Prakash, S., \& Bhandari, B. R. (2016). 3d printing technologies applied for food design: Status and prospects. Journal of Food Engineering. https://doi.org/10.1016/j.jfoodeng.2016.01.025

Guerrero, P., Beatty, E., Kerry, J. P., \& De La Caba, K. (2012). Extrusion of soy protein with gelatin and sugars at low moisture content. Journal of Food Engineering, 110(1), 53-59. https://doi.org/10.1016/j.jfoodeng.2011.12.009

Hao, L., Mellor, S., Seaman, O., Henderson, J., Sewell, N., \& Sloan, M. (2010). Material characterisation and process development for chocolate additive layer manufacturing. Virtual and Physical Prototyping, 5(2), 5764. https://doi.org/10.1080/17452751003753212

Hutchings, J. B. (2011). Food Color and Appearance (2nd ed.). Glasgow, UK: Springer. Retrieved from http://www.springer.com/gp/book/9781441951939

Koushki, M., \& Azizi, M. (2015). Effect of Different Formulations on Mechanical and Physical Properties of Calcium Alginate Edible Films. Journal of Food Quality, 2(2), 45-50.

Le Tohic, C., O’Sullivan, J. J., Drapala, K. P., Chartrin, V., Chan, T., Morrison, A. P., ... Kelly, A. L. (2017). Effect of 3D printing on the structure and textural properties of processed cheese. Journal of Food Engineering, 220,56-64 . https://doi.org/10.1016/j.jfoodeng.2017.02.003

Lille, M., Nurmela, A., Nordlund, E., Metsä-Kortelainen, S., \& Sozer, N. (2017). Applicability of protein and fiberrich food materials in extrusion-based 3D printing. Journal of Food Engineering, 220, 20-27 https://doi.org/10.1016/j.jfoodeng.2017.04.034

Lipton, J., Arnold, D., Nigl, F., Lopez, N., Cohen, D., Norén, N., \& Lipson, H. (2010). MUTLI-MATERIAL FOOD PRINTING WITH COMPLEX INTERNAL STRUCTURE SUITABLE FOR CONVENTIONAL POSTPROCESSING. In: 21st Annual International Solid Freeform Fabrication Symposium - an Additive Manufacturing Conference, SFF 2010, 809-815.

Liu, Z., Zhang, M., \& Bhandari, B. (2018). Effect of gums on the rheological, microstructural and extrusion printing characteristics of mashed potatoes. International Journal of Biological Macromolecules, 117, 1179-1187. 
425

426

427

428

429

430

431

432

433

434

435

436

437

438

439

440

441

442

443

444

445

446

447

448

449

Mali, S., Sakanaka, L. S., Yamashita, F., \& Grossmann, M. V. E. (2005). Water sorption and mechanical properties of cassava starch films and their relation to plasticizing effect. Carbohydrate Polymers, 60(3), 283-289. https://doi.org/10.1016/J.CARBPOL.2005.01.003

Milani, J., \& Maleki, G. (2012). Hydrocolloids in Food Industry. In D. B. Valdez (Ed.), Food Industrial ProcessesMethods and Equipment (p. 418). Iran: INTECH. https://doi.org/10.5772/2491

Periard, D., Schaal, N., Schaal, M., Malone, E., \& Lipson, H. (2007). Printing Food. Proceedings of the 18th Solid Freeform Fabrication Symposium, 564-574. https://doi.org/10.1007/s00216-007-1293-0

Phan, T. D., Debeaufort, F., Luu, D., \& Voilley, A. (2005). Functional Properties of Edible Agar-Based and StarchBased Films for Food Quality Preservation. Journal of Agriculture AND Food Chemistry, 53(4), 973-981. https://doi.org/10.1021/JF040309S

Serizawa, R., Shitara, M., Gong, J., Makino, M., Kabir, M. H., \& Furukawa, H. (2014). 3D jet printer of edible gels for food creation. Proceedings of SPIE - The International Society for Optical Engineering, 9058, 1-5. https://doi.org/10.1117/12.2045082

Severini, C., Derossi, A., Ricci, I., Caporizzi, R., \& Fiore, A. (2017). Printing a blend of fruit and vegetables. New advances on critical variables and shelf life of 3D edible objects. Journal of Food Engineering, 220,89-100 https://doi.org/10.1016/j.jfoodeng.2017.08.025

Sharma, M., Kristo, E., Corredig, M., \& Duizer, L. (2017). Effect of hydrocolloid type on texture of pureed carrots: Rheological and sensory measures. Food Hydrocolloids, 63, 478-487. https://doi.org/10.1016/j.foodhyd.2016.09.040

Shi, X., \& BeMiller, J. N. (2002). Effects of food gums on viscosities of starch suspensions during pasting. Carbohydrate Polymers, 50(1), 7-18. https://doi.org/10.1016/S0144-8617(01)00369-1

Simon. (2015). PepsiCo is creating new deep-ridged potato chips on 3D printers |3D Printer News \&amp; 3D Printing News.

Southerland, D., Walters, P., \& Huson, D. (2011). Edible 3D Printing. Digital Fabrication 2011 Conference, NIP 27, 27th International Conference on Digital Printing Technologies, 819-822. 
Souza, A. C., Benze, R., Ferrão, E. S., Ditchfield, C., Coelho, A. C. V, \& Tadini, C. C. (2012). Cassava starch biodegradable films: Influence of glycerol and clay nanoparticles content on tensile and barrier properties and glass transition temperature. LWT - Food Science and Technology, 46(1), 110-117. https://doi.org/10.1016/j.lwt.2011.10.018

Sun, J., Peng, Z., Yan, L., Fuh, J. Y. H., Hong, G. S., H Fuh, J. Y., \& Soon Hong, G. (2015). 3D food printing—An innovative way of mass customization in food fabrication. Journal of Bioprinting, 1(1), 27-38. https://doi.org/10.18063/IJB.2015.01.006

Truong, V. D., \& Walter, W. M. (1994). Physical and Sensory Properties of Sweetpotato Puree Texturized with Cellulose Derivatives. Journal of Food Science, 59(6), 1175-1180.

Truong, V. D., Walter, W. M., JR., \& Giesbrecht, F. G. (1995). texturization and sensory properties of sweet potato puree with alginate. Journal of Food Science, 60(5), 1054-1059.

Wang, L., Zhang, M., Bhandari, B., \& Yang, C. (2017). Investigation on fish surimi gel as promising food material for 3D printing. Journal of Food Engineering, 220, 101-108 https://doi.org/10.1016/j.jfoodeng.2017.02.029

Yang, F., Zhang, M., Bhandari, B., \& Liu, Y. (2018). Investigation on lemon juice gel as food material for 3D printing and optimization of printing parameters. LWT - Food Science and Technology, 87, 67-76. https://doi.org/10.1016/j.lwt.2017.08.054

Yang, Q., Yang, Y., Luo, Z., Xiao, Z., Ren, H., Li, D., \& Yu, J. (2016). Effects of Lecithin Addition on the Properties of Extruded Maize Starch. Journal of Food Processing and Preservation, 40(1), 20-28. https://doi.org/10.1111/jfpp.12579

Zampollo, F., Kniffin, K. M., Wansink, B., \& Shimizu, M. (2012). Food plating preferences of children: The importance of presentation on desire for diversity. Acta Paediatrica, International Journal of Paediatrics, 101(1), 61-66. https://doi.org/10.1111/j.1651-2227.2011.02409.x

Zhao, Q., Zhao, M., Yang, B., \& Cui, C. (2009). Effect of xanthan gum on the physical properties and textural characteristics of whipped cream. Food Chemistry, 116(3), 624-628. https://doi.org/10.1016/j.foodchem.2009.02.079 
477

478

479

480

481

482

483

484

485

486

487

488

489

490

491

492

493

494

495

496

497

498

499

500

501

502

503

504

505

506 
Table 1 Values of Force, weight collected and SME of potato puree extruded at different speeds and hole diameter

\begin{tabular}{|c|c|c|c|c|}
\hline \multicolumn{2}{|c|}{ Extrusion conditions } & \multicolumn{3}{|c|}{ Parameters } \\
\hline Diameter hole & $\begin{array}{l}\text { Speed } \\
\left(\mathrm{mm} \cdot \mathrm{s}^{-1}\right)\end{array}$ & $\begin{array}{c}\text { Force } \\
\left(\mathrm{kg} \cdot \mathrm{ms}^{-2}\right)\end{array}$ & $\begin{array}{l}\text { Weight collected } \\
\text { (g) }\end{array}$ & $\begin{array}{c}\text { SME } \\
\left(\mathrm{KJ}^{\mathrm{kg}}{ }^{-1}\right)\end{array}$ \\
\hline $5 \mathrm{~mm}$ & $\begin{array}{l}1 \\
2 \\
4\end{array}$ & $\begin{array}{c}113.4 \pm 0.6^{\mathrm{a}} \\
86.1 \pm 1.6^{\mathrm{b}} \\
68.1 \pm 1.3^{\mathrm{c}}\end{array}$ & $\begin{array}{l}36.6 \pm 0.3^{\mathrm{a}} \\
35.5 \pm 0.4^{\mathrm{a}} \\
34.0 \pm 1.5^{\mathrm{a}}\end{array}$ & $\begin{array}{l}62.0 \pm 0.2^{\mathrm{a}} \\
48.5 \pm 1.5^{\mathrm{b}} \\
40.1 \pm 1.0^{\mathrm{c}}\end{array}$ \\
\hline $3 \mathbf{m m}$ & $\begin{array}{l}1 \\
2 \\
4\end{array}$ & $\begin{array}{l}198.2 \pm 7.8^{\mathrm{A}} \\
141.9 \pm 2.2^{\mathrm{B}} \\
107.5 \pm 2.7^{\mathrm{C}}\end{array}$ & $\begin{array}{l}35.0 \pm 2.1^{\mathrm{a}, \mathrm{b}} \\
31.0 \pm 0.7^{\mathrm{b}, \mathrm{c}} \\
31.8 \pm 0.7^{\mathrm{b}, \mathrm{c}}\end{array}$ & $\begin{array}{c}120.8 \pm 3.1^{\mathrm{A}} \\
91.7 \pm 3.5^{\mathrm{B}} \\
67.7 \pm 0.1^{\mathrm{C}}\end{array}$ \\
\hline
\end{tabular}

510

511 Values are mean of three replicates \pm standard deviation.

Different letters of Mean values in the same column differ significantly $(\mathrm{P}<0.05)$ (small and 
527 Table 2 Values of extrusion parameters and Specific Mechanical Energy (SME) obtained at 3mm hole

528 diameter and $2 \mathrm{~mm} . \mathrm{s}^{-1}$ speed printer of potato puree with $1 \%$ of different additives

\begin{tabular}{lccc}
\hline Samples & Force applied $\left(\mathrm{kg} \cdot \mathrm{ms}^{-2}\right)$ & Weight collected $(\mathrm{g})$ & SME $\left(\mathrm{kJ} \cdot \mathrm{kg}^{-1}\right)$ \\
\hline potato puree & $141.9 \pm 2.2^{\mathrm{c}}$ & $31.0 \pm 0.7^{\mathrm{b}}$ & $91.7 \pm 3.5^{\mathrm{c}}$ \\
potato puree+1\% alginate & $261.6 \pm 3.8^{\mathrm{b}}$ & $32.2 \pm 1.3^{\mathrm{a}, \mathrm{b}}$ & $162.4 \pm 10.1^{\mathrm{b}}$ \\
potato puree+1\% agar & $332.1 \pm 10.6^{\mathrm{a}}$ & $30.6 \pm 1.0^{\mathrm{a}, \mathrm{b}}$ & $217.0 \pm 0.3^{\mathrm{a}}$ \\
potato puree+1\% glycerol & $82.3 \pm 1.0^{\mathrm{d}}$ & $31.7 \pm 0.5^{\mathrm{a}, \mathrm{b}}$ & $51.9 \pm 2.1^{\mathrm{d}}$ \\
potato puree+1\% lecithin & $86.5 \pm 2.8^{\mathrm{e}}$ & $33.3 \pm 0.8^{\mathrm{a}}$ & $52.0 \pm 0.6^{\mathrm{d}}$ \\
\hline
\end{tabular}

529

530

531 Values are mean of three replicates \pm standard deviation.

532 Different letters of Mean values in the same column (corresponding to the same parameter) differ

533 significantly $(\mathrm{P}<0.05$

534

535

536

537

538

539

540

541

542

543

544

545

546

547

548 
Table 3 Values of Mechanical Characteristics: firmness, consistency and cohesiveness of additives at 0.5,1 and $1.5 \%$ concentration

553

\begin{tabular}{|c|c|c|c|c|}
\hline Additive & Concentration (\%) & Firmness (g) & Consistency (g.s) & Cohesiveness $\left(\mathrm{g}_{554}\right.$ \\
\hline \multirow{3}{*}{ Glycerol } & 0.5 & $15.4 \pm 0.9^{\mathrm{a}}$ & $275.0 \pm 29.2^{\mathrm{a}}$ & $-3.6 \pm 3.0^{\mathrm{a}}$ \\
\hline & 1 & $15.0 \pm 1.4^{\mathrm{a}}$ & $293.8 \pm 36.4^{\mathrm{a}}$ & $-2.4 \pm 0.4^{\mathrm{a}}$ \\
\hline & 1.5 & $13.5 \pm 1.5^{\mathrm{a}}$ & $249.5 \pm 43.1^{\mathrm{a}}$ & $-2.5 \pm 0.9^{\mathrm{a}}$ \\
\hline \multirow{3}{*}{ Lecithin } & 0.5 & $13.9 \pm 0.9^{\mathrm{a}}$ & $255.0 \pm 25.5^{\mathrm{a}}$ & $-3.1 \pm 0.5^{\mathrm{a}}$ \\
\hline & 1 & $14.7 \pm 1.4^{\mathrm{a}}$ & $269.8 \pm 13.5^{\mathrm{a}}$ & $-2.4 \pm 0.4^{\mathrm{a}}$ \\
\hline & 1.5 & $14.2 \pm 0.9^{\mathrm{a}}$ & $275.2 \pm 29.2^{\mathrm{a}}$ & $-2.3 \pm 0.6^{\mathrm{a}}$ \\
\hline \multirow{3}{*}{ Agar } & 0.5 & $336.0 \pm 54.9^{b}$ & $2347.0 \pm 699.5^{b}$ & $-74.0 \pm 36.5^{\mathrm{b}}$ \\
\hline & 1 & $1202.3 \pm 158.8^{\mathrm{c}}$ & $11858.7 \pm 417.5^{\mathrm{c}}$ & $-245.3 \pm 31.7^{\mathrm{c}}$ \\
\hline & 1.5 & $5864.7 \pm 193.6^{\mathrm{d}}$ & $55070.0 \pm 1714.5^{\mathrm{d}}$ & $-687.7 \pm 86.5^{\mathrm{d}}$ \\
\hline \multirow{3}{*}{ Alginate } & 0.5 & $13.5 \pm 1.3^{\mathrm{a}}$ & $204.2 \pm 50.0^{\mathrm{a}}$ & $-3.4 \pm 0.5^{\mathrm{a}}$ \\
\hline & 1 & $16.0 \pm 0.8^{\mathrm{a}}$ & $313.7 \pm 34.5^{\mathrm{a}}$ & $-4.3 \pm 0.5^{\mathrm{a}}$ \\
\hline & 1.5 & $15.3 \pm 0.6^{\mathrm{a}}$ & $291.2 \pm 13.2^{\mathrm{a}}$ & $-2.7 \pm 0.4^{\mathrm{a}}$ \\
\hline
\end{tabular}

deviation.

569 Different letters of Mean values in the same column (corresponding to the same parameter) differ significantly 
Table 4 Firmness values $(\mathrm{kPa})$ of potato puree samples with glycerol, lecithin, agar and alginate at $0.5,1$ and $1.5 \%$ concentration

588

589

590

591

592

593

594

595

596

597

598

599

600

601

602

603

604 605

606

607

608

609

610

611

612

613

614

8

\begin{tabular}{c|cc}
\hline \multicolumn{1}{c}{ Sample } & Concentration (\%) & Firmness (kPa) \\
\hline \multirow{2}{*}{ Potato puree +Alginate } & 0.5 & $10.0^{\mathrm{c}}$ \\
& 1 & $13.2^{\mathrm{b}, \mathrm{c}}$ \\
\hline Potato puree +Agar & 1.5 & $22.7^{\mathrm{a}}$ \\
\hline \multirow{3}{*}{ Potato puree +Lecithin } & 0.5 & $10.9^{\mathrm{c}}$ \\
& 1 & $17.1^{\mathrm{b}}$ \\
& 1.5 & $25.8^{\mathrm{a}}$ \\
\hline Potato puree +Glycerol & 0.5 & $4.3^{\mathrm{d}}$ \\
& 1.5 & $4.4^{\mathrm{d}}$ \\
& 0.5 & $3.6^{\mathrm{d}}$ \\
\hline Potato puree & 1.5 & $5.0^{\mathrm{d}}$ \\
\hline
\end{tabular}

Values are mean of three replicates

Different letters with Mean values in the same column (corresponding to the same parameter) differ significantly $(\mathrm{P}<0.05)$ 

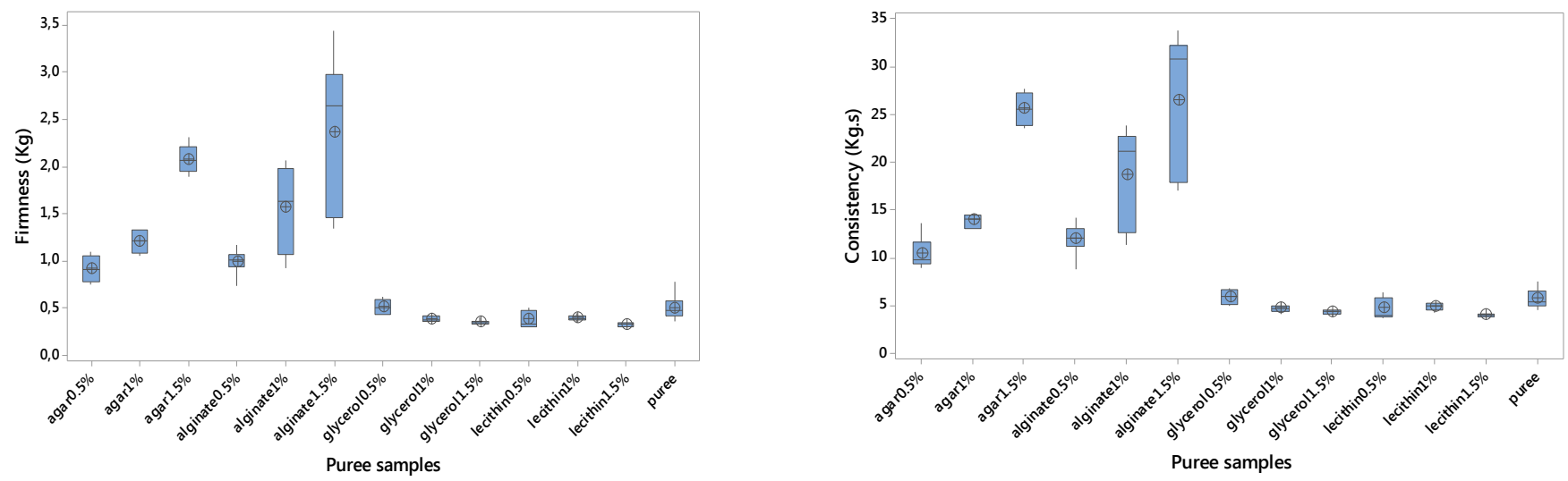

616

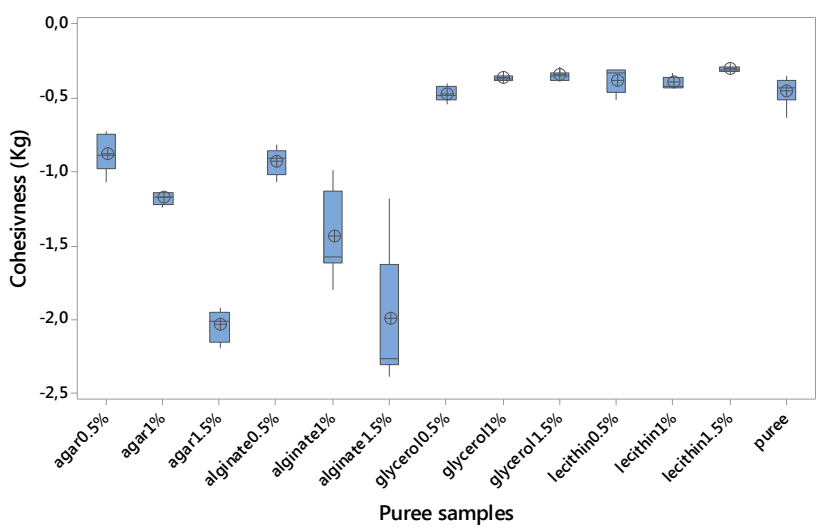

625

626

627

Fig.1 Box plot analysis of the mechanical characteristics firmness (a), consistency (b) and cohesiveness (c) of potato puree and potato puree with agar, alginate, glycerol and lecithin at concentration of $0.5,1$ and $1.5 \%$.

629

630

631 

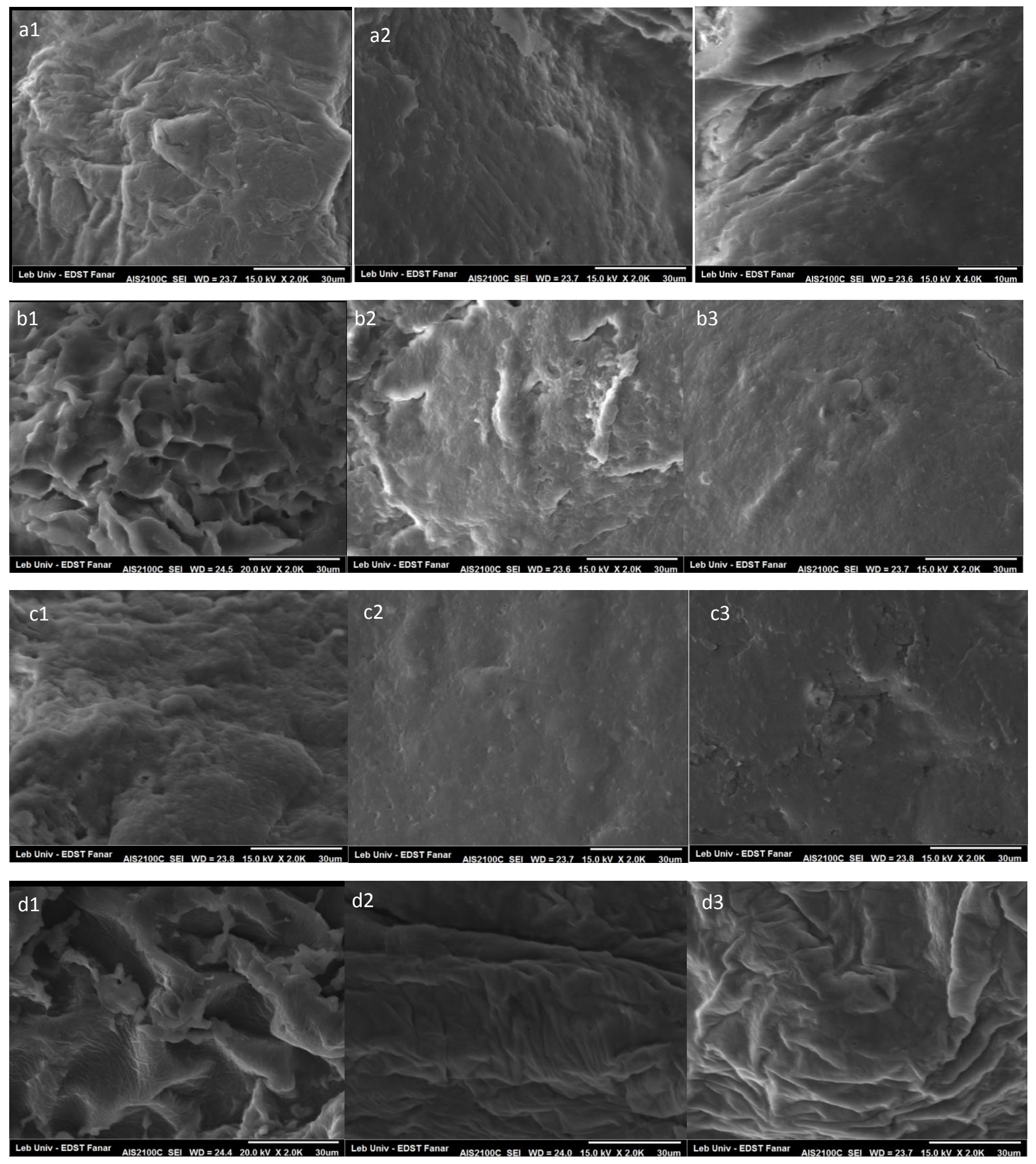

637 alginate; $b 1, \mathrm{~b} 2, \mathrm{~b} 3 \mathrm{~b}$ potato puree with glycerol; $\mathrm{c} 1, \mathrm{c} 2, \mathrm{c} 3$ potato puree with lecithin; d1, d2, d3 potato 638 puree with agar. *arrows correspond to pores formation within lecithin. 

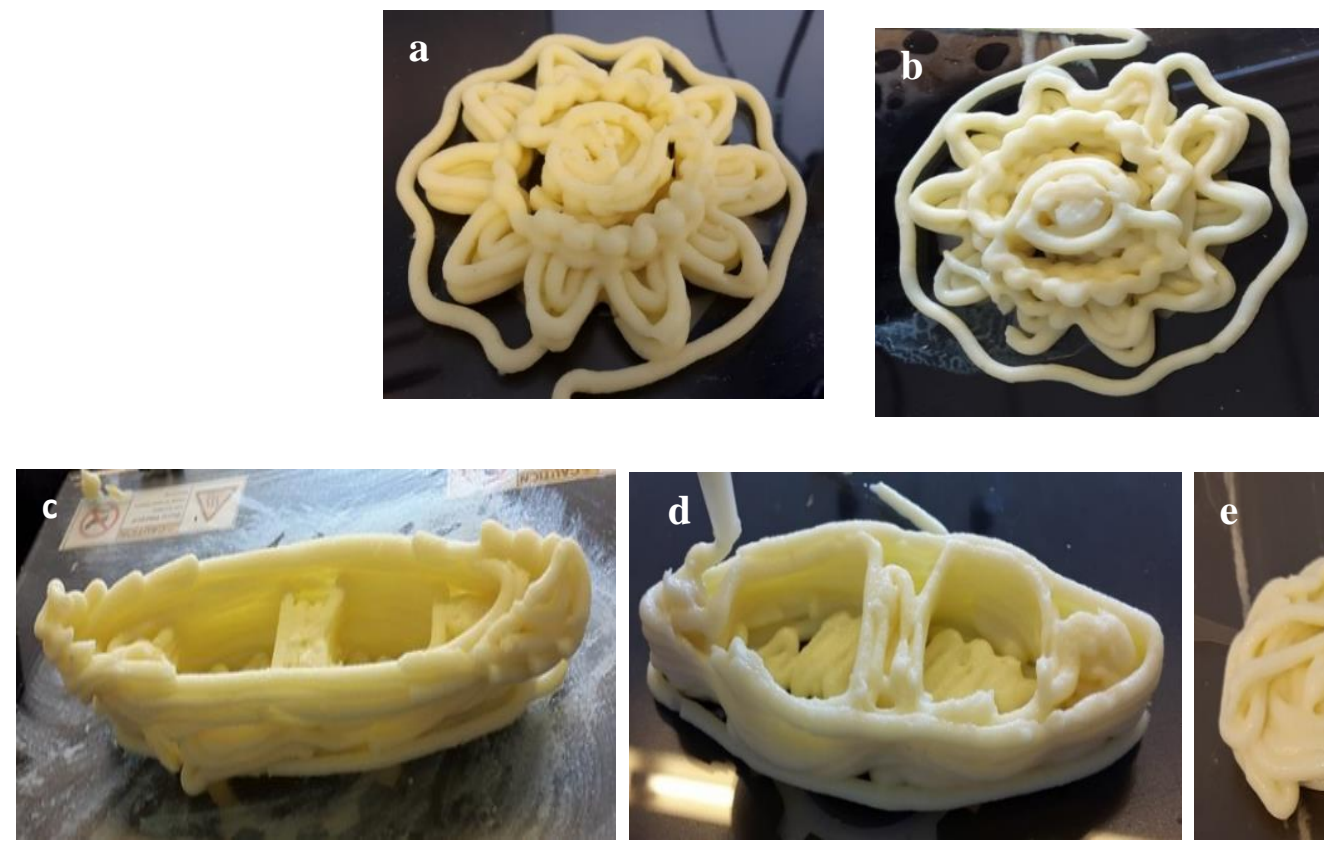

655

656

Fig. 3 The influence of the substrate and shape design on 3D printed products of potato puree alone or with additives when is extruded at $4 \mathrm{~mm}$ nozzle. Fig 3(a, b) Influence of substrate printed : (a) potato
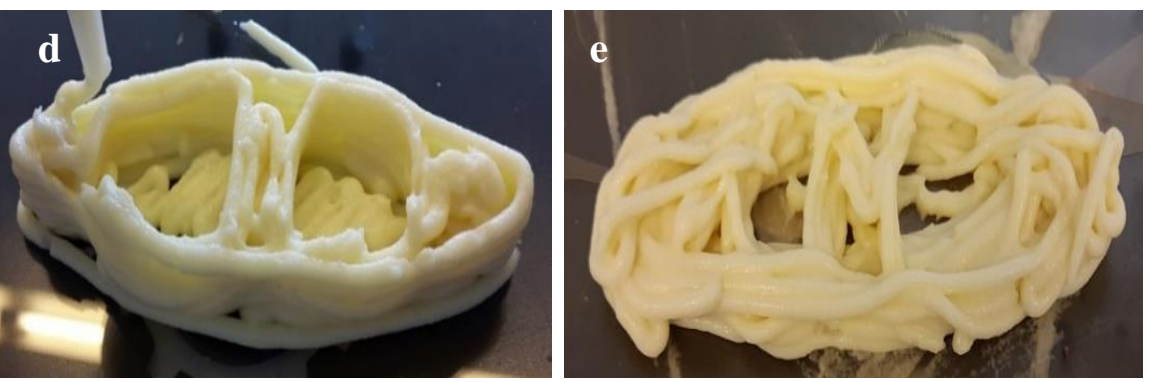

659 puree with $1 \%$ alginate, (d) potato puree alone at primary stages of printing and (e) potato puree alone at final stages of printing.

661

662

663

664

665

666

667

668 


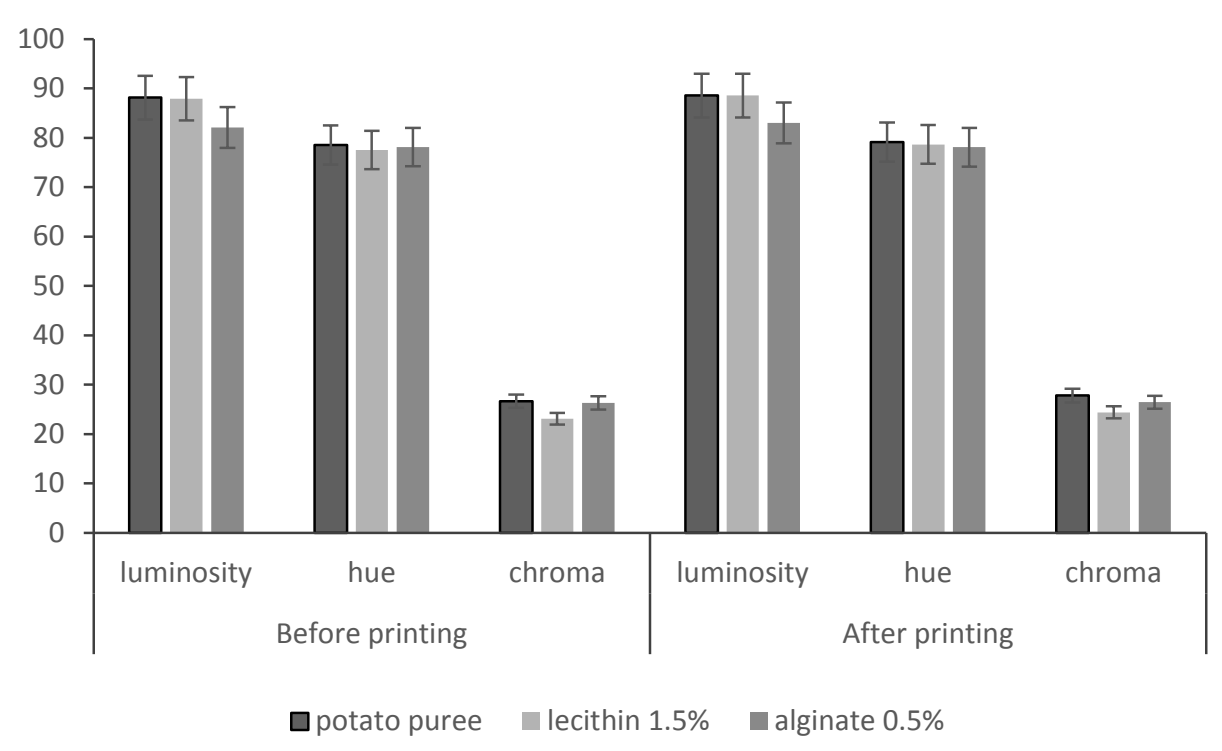

670

671

672 Fig. 4 Values of Luminosity, Chroma and Hue angle in color surface of potato puree alone, potato puree 673 with $1.5 \%$ lecithin and potato puree with $0.5 \%$ alginate before and after $3 \mathrm{D}$ printing. Values are mean \pm 674 standard deviation $(\mathrm{n}=6)$

675

676

677

678

679

680

681

682

683

684 